\title{
BMJ Open Quality STI initiative: Improving testing for sexually transmitted infections in women
} Ryan Christopher Chadwick, ${ }^{1}$ Kathleen McGregor, ${ }^{1}$ Paula Sneath, ${ }^{1}$
Joshua Rempel, ${ }^{1}$ Betty Li Qun He, ${ }^{1}$ Allison Brown, ${ }^{1,2}$ Grant Seifred, ${ }^{3}$
John McAuley, ${ }^{1,3}$ Ralph John Kamatovic, ${ }^{4}$ Muhanad Al-Husari, ${ }^{1,3}$ Salim Ahmed, ${ }^{3}$
Monica Bertolo, ${ }^{1,3}$ Doug Munkley, ${ }^{5}$ Maynard Luterman ${ }^{1,3}$

\section{ABSTRACT}

Canadian urgent care and walk-in medical clinics provide health care for a population that may be poorly covered by traditional health care structures. Despite evidence suggesting that women with urinary complaints experience a high incidence of sexually transmitted infections (STIs), this population may be under-tested in this particular setting. The aim of this quality improvement initiative was to increase STI testing in women presenting with GU complaints. Implementation of an opt-out method of STI testing for women ages 16 and older was introduced at three walk-in clinics. Women presenting with GU complaints were given the opportunity to provide samples for both conventional urine culture and nucleic acid amplification testing (NAAT) for non-viral STIs. Patients received treatment according to standard of care and public health was notified as per local regulations. Testing rate and STI incidence was tracked via clinic electronic medical records (EMRs). Overall results were tracked using run charts and compared to historical data for the year prior to the start of the project. Over a 1 year period prior to this intervention, only $65 \mathrm{STI}$ tests were performed in over 1100 GU complaints (5.5\%). Six STIs were identified during this time. During the 36 -week project period, testing increased to $45 \%$ of the patient population (320/707). The STI detected incidence increased from $0.51 \%$ to $1.4 \%$ in all women, and from $0.84 \%$ to $3.4 \%$ in women aged 16-29 years. An opt-out method was an effective intervention for increasing STI testing within the walk-in clinic setting. With optimisation, significant increases in testing rates can be obtained without substantially increasing clinic workload and at no economic cost to the clinic. As expected, detected incidence rates of STIs were higher than the recognised population prevalence.

\section{PROBLEM}

Incidence rates of bacterial STIs have been increasing in North America over the last two decades. $^{1-3}$ This is highly concerning as bacterial STIs (ie, chlamydia and gonorrhoea) are responsible for substantial morbidity and mortality due to their causal role in pelvic inflammatory disease (PID) and ectopic pregnancy. ${ }^{3}$ While curable, infection with bacterial STIs also sharply increases the risk of HIV transmission, a devastating infection. ${ }^{3}$ In Canada, reported incidence rates of chlamydia has increased by approximately $200 \%$ since its low point in 1996. In the same period, the gonorrhoea incidence rate climbed by $225 \%$, and syphilis, once all but eradicated, increased by $550 \%{ }^{2}$ STI rates in the USA have also been steadily increasing over the last two decades and are now at record levels. ${ }^{3}$

This quality improvement initiative was inspired by several previous studies demonstrating an alarmingly high incidence of STIs in women presenting to emergency departments (EDs) and sexual health clinics with GU complaints. ${ }^{4-6}$ In particular, a research study performed at Case Western University in the USA evaluated all women presenting to a large urban ED with any GU symptoms for STIs. ${ }^{5}$ Patients were included in the study if they reported typical urinary tract infection (UTI) symptoms, such as dysuria, frequency, urgency or haematuria, or if they reported typical STI symptoms, such as vaginal itching, burning or discharge. Patients were first evaluated by the emergency physician using standard practices. All participants subsequently underwent thorough diagnostic testing for UTIs and STIs, regardless of the nature of their GU complaint. This study found that $>20 \%$ of women presenting to the ED with a GU complaint tested positive for a non-viral STI. Importantly, nearly $40 \%$ of these cases were missed during initial evaluation using usual practices.

Initial consideration was given to running this initiative in local EDs; however, the Niagara region has a large and highly accessible network of walk-in clinics staffed by local emergency and general practice physicians. Visits to these clinics are covered by provincially administered universal healthcare. Our initial discussion with local physicians indicated that patients with GU symptoms present 
far more frequently to walk-in clinic settings rather than EDs. Providers working in these walk-in clinics agreed with our assessment and were eager to be involved in the project, leading to a staggered launch of our initiative across three busy community clinics.

The three clinics participating in the project are located in the Niagara region of Southern Ontario, Canada. Two of the clinics are in Niagara Falls and one in St. Catharines. The two Niagara Falls clinics are in suburban locations (one independent, one in a 'big-box' retailer), while the St. Catharines clinic is located in the city's urban core. Combined, the clinics see a total of approximately 125 000 patients per year.

GU complaints (eg, dysuria, frequency, urgency or haematuria) represent one of the most common health issues among patients seen at these clinics. In the year prior to this intervention, over 1100 visits were related to GU complaints, representing approximately $1 \%$ of the clinics' total annual visits. Despite these numbers, only 65 bacterial STI tests were ordered, covering $\sim 5 \%$ of the GU presentations.

\section{AIM}

The aim of this initiative is to increase the testing rate for non-viral STIs among women with GU complaints from a baseline of $5.5 \%$ to $>50 \%$, within 3 months of implementation in a group of three walk-in clinics in the Niagara region of Ontario, Canada.

\section{BACKGROUND}

Infection with non-viral STIs often presents with vague GU symptoms, such as dysuria and urinary frequency. ${ }^{4}$ Unfortunately, much of the lay population appears unaware of this, and many primary care physicians may not fully appreciate the extent or consequences of this knowledge gap. As such, both physicians and patients often consider infection with non-viral STIs, such as Chlamydia trachomatis (CT), Neisseria gonorrhoea (NG) and Trichomonas vaginalis (TV), only in the setting of classic STI symptoms, such as vaginal/penile discharge or burning. ${ }^{4}$ Failing to identify infections that present atypically creates an important quality gap in patient care. STIs are not only bothersome to the patient, but untreated infections can pose devastating risks for women. PID is one long-term consequence of these infections and can carry life-altering morbidity including infertility, chronic pain and ectopic pregnancy. ${ }^{7-10}$ In addition, risk of co-infection with HIV and HCV is much higher in those infected with non-viral STIs. ${ }^{11} 12$

Recently, the reported prevalence of non-viral STIs has risen sharply in the high-income countries, including in Canada. ${ }^{13}$ In Canada, CT and NG infections are reportable to public health authorities. Current reports suggest incidence rates of approximately 300 per 100000 and 33 per 100 000, respectively. Reports of CT incidence across the Niagara Region in Canada are similar to the national average $(250 / 100000) \cdot{ }^{13-15}$ The highest rates are among young women. ${ }^{16}$ Trichomoniasis is not a reportable infection in Canada, nor with Ontario provincial or Niagara municipal health agencies, thus the local incidence is unknown. It is evident that STIs continue to persist in the population despite well-understood transmission and effective treatments. ${ }^{15}$ In addition, as current Canadian Taskforce on Preventative Healthcare guidelines (introduced in 2013) recommend Pap screening every threeyears beginning at age 25 years, this screening is no longer routine for younger women. ${ }^{17}$ Primary care providers and university/college clinics often screen younger women, but these resources may not be accessible or available to all women.

\section{MEASUREMENT}

Our primary outcome measure was overall STI testing rate, defined as the fraction of women with GU complaints who received STI testing. Using the clinics' EMRs, baseline STI testing rates were determined for the previous year (1 October 2015 to 30 September 2016), and then the number of tests performed on a biweekly basis were tracked prospectively following implementation of the intervention. In addition, testing rates were tracked for the subgroup of women aged 16-29 years, as this population has been shown to be at increased risk for STIs. ${ }^{13} 16$ Previous STI testing was rare at the participating clinics and the physicians did not generally consider STIs on their differential diagnosis for GU complaints. There was no turnover of physicians during the project period. As such, it is extremely likely that the increases in testing rate were attributable only to our interventions.

As secondary outcomes, we calculated the rate of STI detection (\% of patients with a positive STI test), and UTI culture rates using the same tracking methods. This initiative targeted an $80 \%$ overall UTI culture rate for our patient population. For each outcome measure, a run chart was produced of testing rates and STI incidence throughout the project for the overall data divided by clinic, and for the subpopulation aged 16-29 years.

\section{DESIGN}

A situational needs assessment at four regional walk-in clinics highlighted that STI testing has not previously been a priority, and that STI testing was rarely performed prior to our intervention. Clinic physicians were divided on the priority of the project voicing three major concerns: (1) clinic flow and time requirements were perceived as significant barriers to participation and strategies were developed to minimise added workload for the physicians and administrative staff as described below; (2) some providers were sceptical that STI rates were sufficiently high to warrant additional testing and (3) several physicians were concerned about the time required to counsel patients properly, and the ethical implications of inadequate counselling. Ultimately, the project proceeded with a subset of interested practitioners at three clinics. The fourth clinic declined to participate, 
citing an inability to handle increased workload. The QI team consisted of five medical students responsible for collecting data, a local physician champion affiliated with all of the participating walk-in clinics and an academic QI lead. In addition, two clinic administrators took the role of coordinating the project within the front-of-house clinic staff. Plan-do-study-act (PDSA) cycles were used to guide the design and implementation of the intervention, and were primarily focused on streamlining the process, maintaining clinic flow, improving communication with patients and preventing project burnout to promote sustainability. The initial design of the project focused on improving testing rates via altering physicians' individual practices (i.e., having physicians identify patients to screen), but it was quickly apparent that this was inefficient and would likely be unsustainable over the long term. This manifested in the form of a rapid decline in testing rate, inconsistent ordering practices and negative physician feedback. The first few PDSA cycles were therefore focused on shifting the role of testing to the clinic nursing and administrative staff, who were appropriately positioned to identify patients who presented with GU complaints that would be eligible for STI screening. The student team met monthly to discuss results, assess the implementation of the intervention and design future PDSA cycles to promote uptake and sustainability of the intervention. The participating physicians were apprised of results via regular email updates.

This initiative was exempt from formal ethical review by the Hamilton Integrated Research Ethics Board, as it was considered a quality improvement project, and as per Article 2.5 of the Tri-Council Policy Statement it was exempt from full ethical review. Ethical considerations included patient consent as well as mandatory STI reporting in Ontario. As this is a quality improvement project using standard-of-care testing and no experimental testing, consent for testing was performed by the project physician in their usual fashion. On the request of the project clinicians, information cards were developed to improve patient understanding of the project and expedite physician/patient discussion. The initial information cards (introduced in PDSA 1) and an updated version (PDSA 4) are available in the online supplemental information.

\section{STRATEGY}

PDSA cycles were used to design, test and implement the intervention. ${ }^{18} 19$ The intervention was piloted with a single lead physician to identify any preliminary issues. The first PDSA cycle was implemented after the initial 2 weeks to address two issues: (1) while additional STI testing was being ordered, it was performed sporadically and the specific tests ordered were inconsistent; (2) STI tests by culture (as opposed to NAAT) and for single organisms were still being ordered. As such, our first change in process was to create a standardised, computer-generated lab order sheet to reduce incomplete testing; no further incomplete testing was performed thereafter. In addition, this change improved physician work flow by eliminating the need to fill out testing forms for each patient. The second change in process was the first version of our 'Patient Information Card' (see online supplemental information). This card was introduced to help facilitate an explanation of this Quality Improvement (QI) initiative to the patients, including the testing involved. Feedback from clinic staff and our lead physician indicated an improvement in patient understanding and process flow with this information card.

A second PDSA cycle, performed after 1 month of project implementation, changed testing from 'opt-in' to 'opt-out', in response to feedback from the clinic team. In short, virtually all patients agreed to testing; however, most urine samples were being precollected (with the pre-existing culture form attached) by clinic staff prior to seeing the physician. This required a second collection if STI testing was to be performed, which was a large detriment to clinic flow. As such, the 'default' testing was changed to collect samples for urinalysis, culture and sensitivity (C\&S) and STI testing. Patients were able to opt-out at two points: by providing a sample for C\&S testing only as per instructions by clinic staff, and more frequently by declining during discussion with the clinic physician (in which case the additional urine sample was discarded). The patient information cards were given to each patient at the time of collection in order to facilitate discussion and provide a brief explanation of the project. At the same time, several other physicians in clinic 1 were brought aboard, and we expanded the project to clinics 2 and 3 .

A third PDSA cycle implemented TV NAAT testing to expand the project scope. TV is not a reportable infection in Ontario and testing laboratories are not required to have NAATs available. We contacted local labs about the availability of NAAT TV testing and while both major regional lab providers responded, only the provider for clinic 3 was able to deliver TV testing. The lab provider for clinics 1 and 2 indicated they intended to offer this service in the future. TV testing was therefore implemented in clinic 3 only. Every STI test at clinic 3 included TV testing after this point; however, as clinic 3 was our smallest clinic, this resulted in the detection of no cases of TV in 36 tests. In short, due to the small number of women tested we were unable to evaluate the utility of TV testing.

A fourth PDSA cycle was focused on patient education. Patient information cards were modified to better explain the concept of public health notification requirements and contact tracing in response to recommendations from our physicians (see online supplemental information). This further facilitated discussion between patients and the physicians involved in the study. Clinic physicians did not report an increase in patient opt out with this intervention.

The first four PDSA cycles were implemented early in the project and these cycles were targeted at optimising 
clinic flow and patient education. A 12-week period of data collection was allowed before implementing our fifth and sixth PDSA cycles. Over this 12-week period, we noticed that testing rates were paradoxically higher among women with urinary symptoms as compared with those with genital tract (GT) symptoms. Surveying the clinic staff indicated that this occurred because urine was often not pre-emptively collected in the case of GT symptoms. It was hypothesised that discussing this with the clinic staff would be sufficient to improve testing rates.

Finally, it was also noted that there was a substantial variation in testing rates among physicians. This was somewhat surprising in a design where STI testing was performed by default. It was also noted that these physicians tended to work the same days of the week, and that these physicians had increased testing rates when covering for other physicians. To address these discrepancies, our final PDSA cycles were implemented in successive biweekly periods. The first of these (PDSA 5-biweekly period 12) was the furnishing of more detailed, personal reports to the clinic physicians. The second of these (PDSA 6) involved QI team members visiting clinics on the appropriate days to remind the clinic staff of the study protocol and address any concerns. A reminder notice was also left behind the desk at each clinic, to help remind clinic staff of the ongoing study. These interventions during biweekly periods 12 and 14 corrected the issues, and improved consistency.

Lastly, in terms of scaling up the project, we added the project physicians and clinics relatively quickly over the first month of the project. In total, three clinics and eight physicians have participated at this point. The time commitment required for data collection limited further scaling of the project. Future efforts to scale up will require additional data collection teams or automated data collection.

\section{RESULTS}

To assess the effectiveness of our intervention, we produced run charts allowing for an ongoing comparison of testing rates with our historical baseline data (figure 1). Run charts tracked both STI and UTI process measures with each data point representing a 2-week period. STI testing rates were tracked separately for each individual clinic, for all clinics combined and for the subset of patients aged 16-29 years. UTI culture rates were tracked similarly.

Overall preintervention and postintervention testing rates and STI incidence were compared. Both testing and detection rates were significantly different before and after implementation (table 1 ). The overall results are presented graphically in figure 2.

Biweekly STI testing rate-all clinics combined

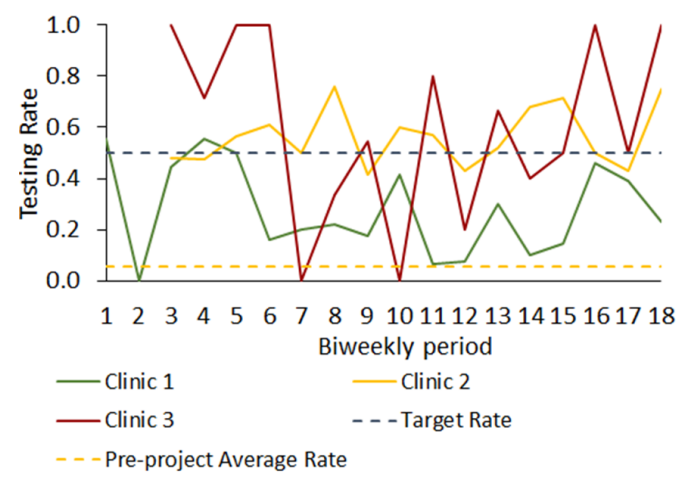

Biweekly STI testing rate-women $<30$-all clinics

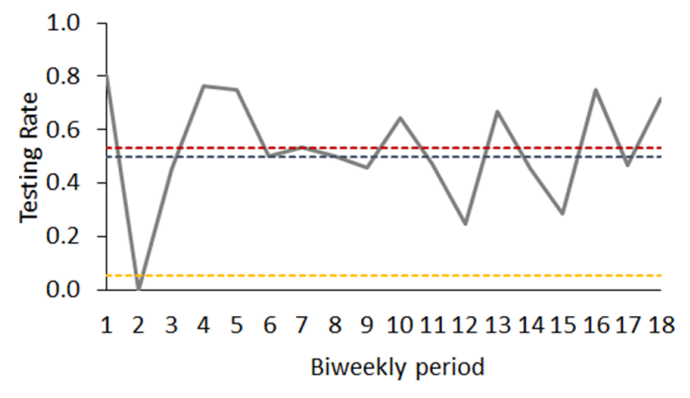

-Testing Rate $\quad$----- Target Rate
---- Retrospective Rate -----. Average Rate
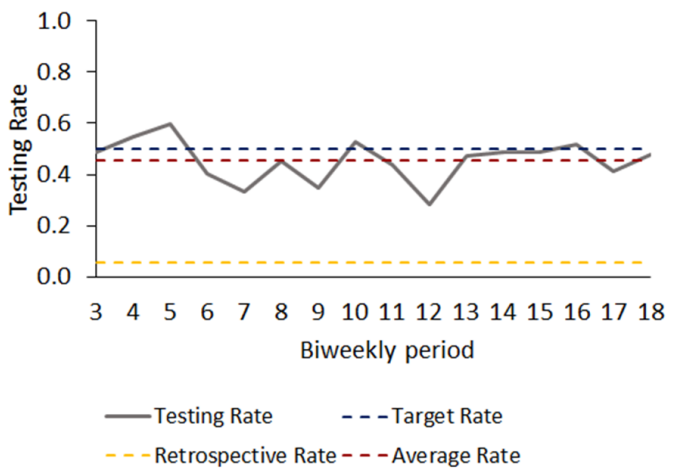

Biweekly UTI testing rate-all clinics

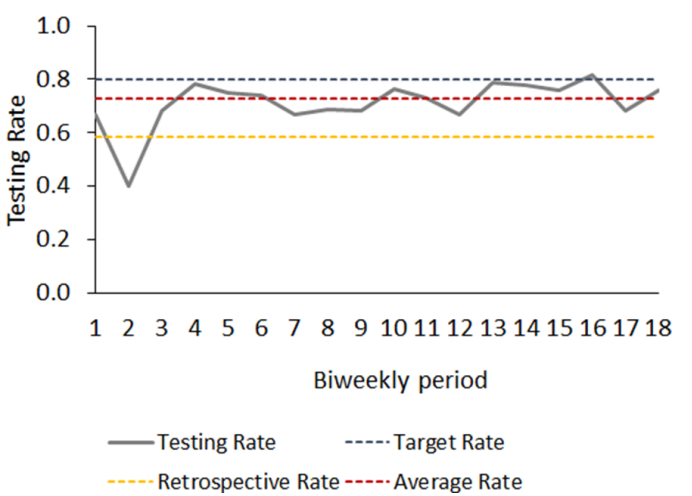


Table 1 STI testing rates, infection rates and comparison of full-year retrospective vs date-matched retrospective data

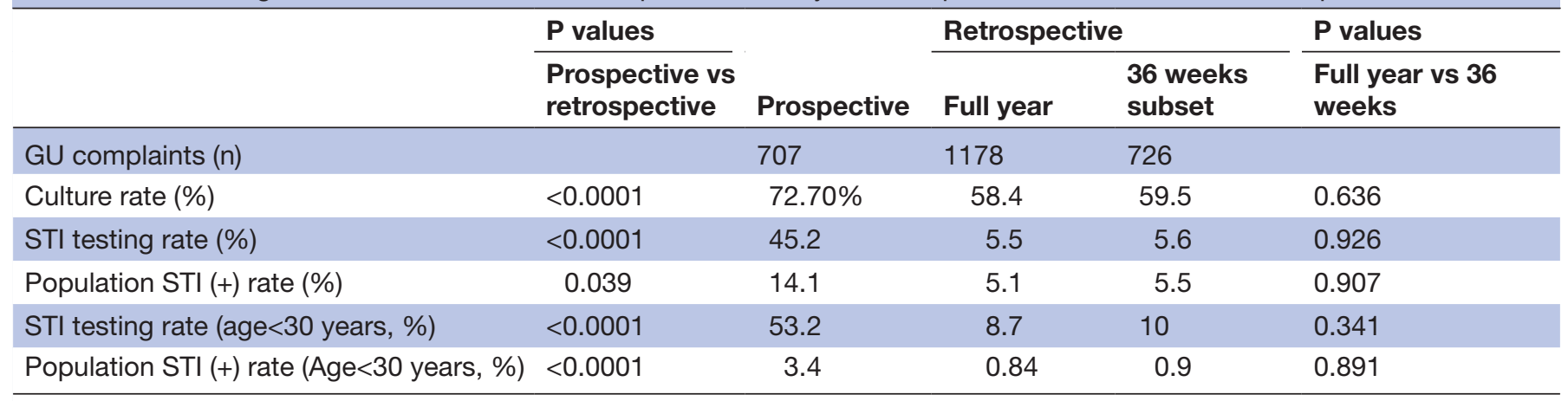

STI, sexually transmitted infections; GU, genitourinary.

The clinics' overall STI testing rate increased from $5.5 \%$ to $45.2 \%$ (effect size: $39.7 \%$, 95\% CI $35.8 \%$ to $43.6 \%, \mathrm{p}<0.0001)$. A similar result was obtained in patients under 30 years of age, where testing increased from $8.6 \%$ to $53.2 \%$ (effect size: $44.6 \%$, 95\% CI $37.4 \%$ to $51.4 \%, \mathrm{p}<0.0001)$. There was a concomitant increase of detected incidence from $0.51 \%$ to $1.4 \%$ (effect size: $0.89 \%, 95 \%$ CI $0.014 \%$ to $2.1 \%, \mathrm{p}<0.041$ ). This increase was $0.84 \%$ to $3.4 \%$ for patients under 30 years (effect size: $2.56 \%, 95 \%$ CI $0.25 \%$ to $5.8 \%, \mathrm{p}<0.024)$. Unsurprisingly, testing efficiency decreased, with the number needed to test to detect one STI increasing from 11 to 32 (95\% CI

Preproject testing rate

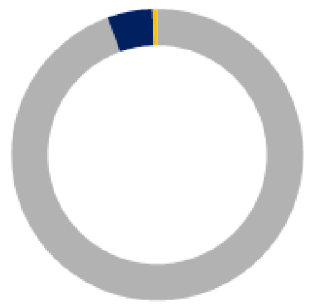

GU complaints untested $(\mathrm{n}=1113,94.5 \%)$

- STI testing negative ( $\mathrm{n}=59,5.0 \%$ )

Positive $(n=6,0.51 \%)$

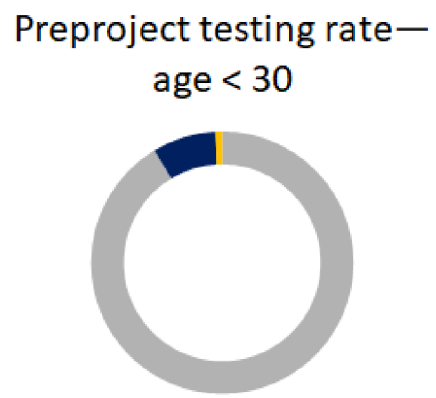

GU complaints untested ( $n=327,91.3 \%$ )

- STI testing negative $(\mathrm{n}=28,7.8 \%)$

Positive ( $n=3,0.84 \%)$
17 to 86 ). Additional testing was more efficient in young women where number needed to test increased from 10 at baseline to 16 after intervention $(95 \% \mathrm{Cl} 8$ to 49$)$.

The enrolment of our clinics and providers was staggered over a period of approximately 3 months. For our historical baseline, we collected data for the 1 year period immediately prior to launching our project. We believe this most accurately represents our providers' long-term practice. However, to ensure seasonal variation did not affect our results, we have compared the full-year data to a subset of the previous year's data that matched each of the providers' project participation dates. There was no

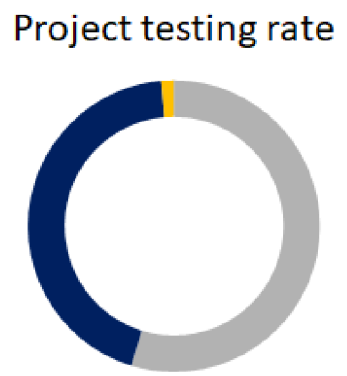

- GU complaints untested ( $\mathrm{n}=387,54.8 \%$ )

- STI testing negative $(n=310,43.8 \%)$

- Positive $(\mathrm{n}=10,1.4 \%)$

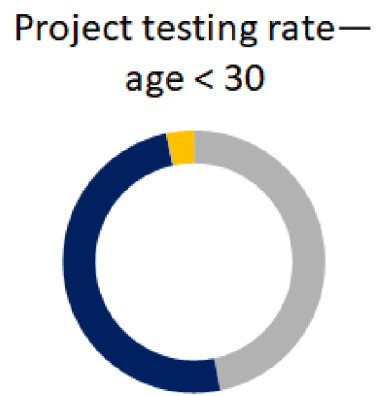

- GU complaints untested ( $\mathrm{n}=109,46.8 \%$ )

n STI testing negative $(\mathrm{n}=116,49.8 \%)$

Positive $(n=8,3.4 \%)$

Figure 2 Comparison of preproject and periproject testing and detection rates for sexually transmitted infections (STIs). GU, genitourinary. 
significant difference between this subset and the fullyear results (table 1 ).

After the initial implementation, the first two PDSA cycles focused on developing our ordering process. After these two cycles, testing rates remained near target for most of the duration of the project. We were unable to fully implement TV testing in PDSA cycle 3 as discussed above. As we were able to quickly achieve satisfactory testing rates, the focus of the fourth PDSA cycle was improved clarity around the patient information card in response to provider concerns. Testing rates dropped substantially around the seventh biweekly cycle, which corresponded with the clinics reopening after the 2016/2017 holiday period. This did not immediately correct in the two periods following, and after a 4-week data collection lag time, two PDSA cycles (PDSA 5 and 6) were implemented. The first, in biweekly period 12 , was a more detailed report to all project physicians to refamiliarise them with the project. The second centred around the knowledge that testing rate varied substantially by day of the week (see the Design section on PDSA cycles). An informal retraining (during biweekly period 14) was conducted for the existing and new clinic staff, and a reminder notice was posted for clinic staff in each clinic to improve consistency among front of house staff. These cycles corresponded with the improved testing rates and consistency after period 12-14 at clinics 2 and 3 .

\section{LESSONS AND LIMITATIONS}

The aim of the project was to increase the rate of non-viral STI testing in walk-in urgent care clinics in the Niagara region, Ontario, Canada. Walk-in clinics represent an ideal location to detect and treat these infections due to their high volume of patients and accessibility within the region. They care for several population groups that are more likely to have undetected STIs including younger patients, patients without access to a regular family doctor and patients with acute GU complaints.

The walk-in clinic environment presents several challenges to changing practice. A different physician works each day and the clinics have a high rate of staff turnover, multiplying the effort required to implement change. As such, clinic flow is of critical importance, and interventions that negatively affect patient throughput are likely to be abandoned or ignored. Finally, it has been our experience that the patient population in the urgent care setting more often demands immediate treatment and is more sceptical of testing and repeat visits than in a general practice setting. With these challenges in mind, a primary strength of this project was its simplicity. It required no major changes in clinic infrastructure or practice patterns and was entirely without economic cost from the clinic's standpoint. Anonymous data collection via EMR searches was straightforward and represented a minimally time-consuming task.

However, the same challenges also led to the project's limitations. In general, early PDSA cycles focused on perceived clinic flow and the physicians' and clinic staff's subjective experience. This led to a measurement issue, as assessment of 'flow' was entirely subjective and informal. Regarding sustainability, the distributed nature of the project adds some difficulty. For each new physician there was an adjustment period, but this was well-defined and organised.

An additional limitation is in our availability of testing. TV is well-accepted as a human pathogen and STI. Unfortunately, effective NAAT testing was not widely available at all labs involved in our study. It is our hope that this will be available in the near future, and we have begun discussions with local labs to facilitate this. This problem is even more pronounced with more recently identified sexually transmitted pathogens. While Ureaplamsa urealyticum and Mycoplasma genitalia have been receiving substantial attention as causes of GU symptoms and morbidity, ${ }^{20-23}$ testing for these pathogens is currently expensive, difficult to access and, in the case of $U$. urealyticum, not paid for by the Ontario health system. As such, broad-based screening in a walk-in clinic setting is not currently viable, despite demonstrated prevalence of up to $5 \% .{ }^{23}$ Nonetheless, targeted screening may be of benefit, especially in the subpopulation of patients with recurrent GU symptoms with negative urine cultures and negative TV, CT and NG NAATs.

With further regard to cost, although the project was essentially free from the clinics' standpoint, there was increased testing which translates into increased cost for the provincially funded health system. Physicians are expected to be stewards of health funding and in order to maximise sustainability we would ideally minimise extraneous testing. We have identified two areas where cost can be reduced. (1) Avoiding double testing: it was commonplace for patients experiencing recurrent UTI symptoms to receive multiple STI tests in a very short window of time (weeks). This represents substantial unnecessary testing. (2) Considering age-based testing: The clear majority of our detected STIs are in a younger population and the morbidities of STIs (infertility, risk of ectopic pregnancy) are also greatest in these patients. It remains unclear if defaulting to screening represents the most cost-effective option in older patients.

\section{CONCLUSION}

The detection and management of STIs remains an important problem that affects health systems worldwide. Current Canadian strategies seem focused on detection efforts through the offices of gynaecologists, at educational institutions and through sexual health clinics. These avenues are not fully effective as evidenced by rising non-viral STI rates. This is partly because these mechanisms are insufficient to reach all patient populations.

GU complaints are well-correlated with STIs. In some North American health jurisdictions, the rates of STIs in the walk-in or ED patient with GU complaints approach or exceed $20 \%{ }^{5}$ The clinics in this study are in suburban, middle-income areas with government-funded healthcare 
and did not have such notable rates. Nonetheless, despite testing rates of $<50 \%$, our clinic population had detected STI incidence rates that are substantially above the reported rates of the population as a whole $(1.7 \%$ vs $0.3 \%)$. Incidence was particularly high in the population aged $16-29$ years $(3.1 \%)$.

The brief nature of walk-in clinic encounters and the relative infrequency of STI detection events had resulted in a practice pattern in our clinics where STI testing was very uncommon, essentially only occurring in the context of an unprompted direct request from a patient. The primary purpose of this project was to drive a sustainable increase in testing rates in the targeted clinics, the results show that the project was a success. Testing rates increased from $5.5 \%$ to $45.2 \%$, nearly meeting the initial target of $50 \%$ and with a concomitant tripling in STI detection. Clinic flow was unhampered by testing and there was no increase in cost to the clinic or its providers. Compliance was optimised by repeated training interventions with new clinic staff, identifying days of reduced testing and subsequently implementing strategies to rectify. While integrated automatic testing of all UTI samples with NAAT yielded higher testing rates, it is not clear whether this testing was cost-effective in all populations. Future strategies to refine automatic testing could increase diagnostic sensitivity and specificity by eliminating low-risk women.

Acknowledgements The authors would like to thank all the hardworking medical and administrative staff in each of the clinics involved in this project. Without their help, this project would not have been possible.

Contributors RCC initiated the project, oversaw data collection and performed the literature review, data interpretation and manuscript preparation. KMG, PS, $\mathrm{JR}$ and $\mathrm{LQH}$ performed data collection and assisted with data interpretation. They also liaised with and trained clinic staff. $A B$ assisted with project design, data interpretation and manuscript preparation. GS, JMA, RJK, MA-H, SA, MB and DM were the clinic physicians who implemented the Ql procedures in the project's walk-in clinics. ML was the lead physician on the project. He contributed to project design, liaised with the data collection team and clinic staff and piloted all interventions.

Funding This project received funding from the Michael DeGroote School of Medicine Niagara Regional Campus in the form of a Student Research Funding Grant (US\$500).

Competing interests None declared.

Patient consent Not required.

Provenance and peer review Not commissioned; externally peer reviewed.

Open access This is an open access article distributed in accordance with the Creative Commons Attribution Non Commercial (CC BY-NC 4.0) license, which permits others to distribute, remix, adapt, build upon this work non-commercially, and license their derivative works on different terms, provided the original work is properly cited, appropriate credit is given, any changes made indicated, and the use is non-commercial. See: http:// creativecommons.org/licenses/by-nc/4.0/.

\section{REFERENCES}

1. Government of Canada. Report on sexually transmitted infections in Canada: 2013-2014. Ottawa, 2017.

2. Public Health Agency of Canada, 2016. Reported cases from 1924 to 2015 in Canada - Notifiable diseases on-line. http://diseases.canada. $\mathrm{ca} /$ notifiable/charts?c=pl

3. Braxton J, Davis D, Flagg E, et al. Sexually transmitted disease surveillance 2016. Atlanta, 2017.

4. Wilbanks MD, Galbraith JW, Geisler WM. Dysuria in the emergency department: missed diagnosis of Chlamydia trachomatis. West $J$ Emerg Med 2014;15:227-30.

5. Tomas ME, Getman D, Donskey CJ, et al. Overdiagnosis of urinary tract infection and underdiagnosis of sexually transmitted infection in adult women presenting to an emergency department. J Clin Microbiol 2015;53:2686-92.

6. Shapiro T, Dalton M, Hammock J, et al. The prevalence of urinary tract infections and sexually transmitted disease in women with symptoms of a simple urinary tract infection stratified by low colony count criteria. Acad Emerg Med 2005;12:38-44.

7. MacDonald NE, Brunham R. The effects of undetected and untreated sexually transmitted diseases: pelvic inflammatory disease and ectopic pregnancy in Canada. Can J Hum Sex 1997;6:161-71.

8. Wiesenfeld HC, Hillier SL, Meyn LA, et al. Subclinical pelvic inflammatory disease and infertility. Obstet Gynecol 2012;120:37-43.

9. Haggerty CL, Gottlieb SL, Taylor BD, et al. Risk of Sequelae after Chlamydia trachomatis Genital Infection in Women. J Infect Dis 2010;201:134-55.

10. Pellati D, Mylonakis I, Bertoloni G, et al. Genital tract infections and infertility. Eur J Obstet Gynecol Reprod Biol 2008;140:3-11.

11. Mayer KH, Venkatesh KK. Interactions of HIV, other sexually transmitted diseases, and genital tract inflammation facilitating local pathogen transmission and acquisition. Am J Reprod Immunol 2011;65:308-16.

12. Terrault NA. Sexual activity as a risk factor for hepatitis $C$. Hepatology 2002;36:S99-105.

13. Government of Canada. The Chief Public Health Officer's report on the state of public health in Canada, 2013 - Infectious disease-The Never-ending Threat. Ottawa, 2013.

14. Niagara Region Health, 2017. Chlamydia - Statistics and incidence rate. https://www.niagararegion.ca/health/statistics/disease/ chlamydia.aspx

15. Satterwhite CL, Gottlieb SL, Romaguera R. CDC Grand rounds: chlamydia prevention: challenges and strategies for reducing disease gurden and sequelae. MMWR Morb Mortal Wkly Rep 2011;60:365-9.

16. Totten S, MacLean R, Payne E, et al. Chlamydia and lymphogranuloma verereum in Canada: 2003-2012 summary report. Ottawa, 2015.

17. Dickinson J, Tsakonas E, Gorber S, et al. Recommendations on screening for cervical cancer. Can Med Assoc J 2013;185:35-45.

18. Taylor MJ, McNicholas C, Nicolay C, et al. Systematic review of the application of the plan-do-study-act method to improve quality in healthcare. BMJ Qual Saf 2014;23:290-8.

19. Leis JA, Shojania KG. A primer on PDSA: executing plan-dostudy-act cycles in practice, not just in name. BMJ Qual Saf 2017;2626:572-7.

20. McGowin CL, Anderson-Smits C. Mycoplasma genitalium: an emerging cause of sexually transmitted disease in women. PLOS Pathog 2011;7:e1001324.

21. Manhart LE, Mostad SB, Baeten JM, et al. High Mycoplasma genitalium organism burden is associated with shedding of HIV-1 DNA from the cervix. $J$ Infect Dis 2008;197:733-6.

22. Perez G, Skurnick JH, Denny TN, et al. Herpes simplex type II and Mycoplasma genitalium as risk factors for heterosexual HIV transmission: report from the heterosexual HIV transmission study. Int J Infect Dis 1998;3:5-11.

23. Gesink D, Racey CS, Seah C, et al. Mycoplasma genitalium in Toronto, Ont: estimates of prevalence and macrolide resistance. Can Fam Physician 2016;62:e96-101. 